
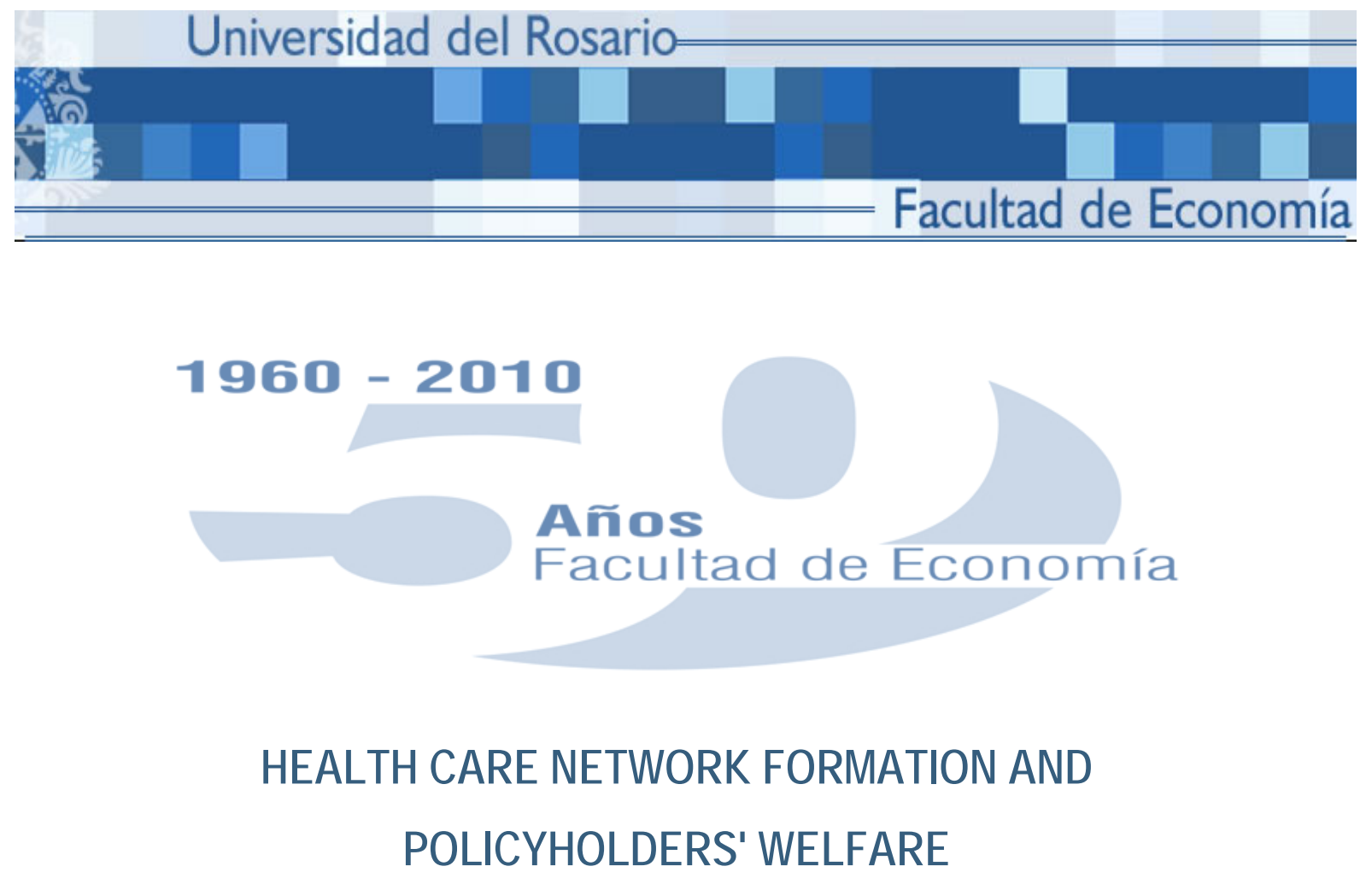

David Bardey

Jean-Marc Bourgeon

SERIE DOCUMENIOS DE TRABAJ $O$

No. 88

Agosto 2010 


\title{
Health Care Network Formation and Policyholders' Welfare*
}

\author{
David Bardey ${ }^{\dagger}$ and Jean-Marc Bourgeon ${ }^{\ddagger}$
}

August 11, 2010

\begin{abstract}
We develop a model in which two insurers and two health care providers compete for a fixed mass of policyholders. Insurers compete in premium and offer coverage against financial consequences of health risk. They have the possibility to sign agreements with providers to establish a health care network. Providers, partially altruistic, are horizontally differentiated with respect to their physical address. They choose the health care quality and compete in price. First, we show that policyholders are better off under a competition between conventional insurance rather than under a competition between integrated insurers (Managed Care Organizations). Second, we reveal that the competition between a conventional insurer and a Managed Care Organization (MCO) leads to a similar equilibrium than the competition between two MCOs characterized by a different objective i.e. private versus mutual. Third, we point out that the ex ante providers' horizontal differentiation leads to an exclusionary equilibrium in which both insurers select one distinct provider. This result is in sharp contrast with frameworks that introduce the concept of option value to model the (ex post) horizontal differentiation between providers.
\end{abstract}

Keywords: Health Care Network, Horizontal Differentiation, Health Care Quality.

Jel Code: I11-L14-L11-L42.

${ }^{*}$ We gratefully acknowledge financial support from the Fondation du Risque (Chaire Santé, Risque et Assurance, Allianz).

${ }^{\dagger}$ Toulouse School of Economics (GREMAQ) and University of Rosario (Bogota).

$¥$ INRA and Ecole Polytechnique. Address: INRA - Économie Publique, 16 rue Claude Bernard, 75231 Paris Cedex 05, FRANCE. E-mail: bourgeon@inapg.fr 


\section{Introduction}

Health care markets are characterized by several market failures (Arrow, 1963). As pointed out in Ma and Riordan (2002), some institutions have emerged as a response to this issue. Vertical integration, or more generally, vertical agreements between insurers and health care providers, are known to be an efficient response to incentives problems. Indeed, additionally to the standard argument of transaction costs reduction, by putting more incentives on the providers' side, managed care organizations (MCOs in the following) also allow to ease the tradeoff between risk spreading and copayments on the policyholders' side (Ma and McGuire, 1998). However, the formation of health care networks in a strategic environment remains misunderstood and the MCOs' impact on policyholders' welfare is still in debate. As has been observed that MCOs' plans are cheaper than conventional insurance, several explanations are given to understand this stylized fact. On one hand, Baker and Corts (1996) and Baranes and Bardey (2006) show that MCOs may benefit from a favorable risk segmentation. On the other hand, some empirical results dealing with health care quality delivered in MCOs reveal that, on average, the quality perceived is lower in MCOs than with conventional insurers (Blendon et al., 1998).

There are two goals to this paper. First, we shed light on this quality-price trade-off issue so as to establish a comparison, expressed in terms of policyholders' welfare, between different market structures observed in the health care sector. Second, we provide a positive argument to explain the formation of health care networks. For this purpose, we consider a set-up in which two insurers and two providers are present and compete for a fixed mass of policy holders. Insurers compete in premium and offer coverage against financial consequences of health risk. They have the possibility to sign agreements with providers to establish a network of health care. We consider that providers, who are partially altruistic, are horizontally differentiated. More precisely, they are characterized by different physical addresses. They choose health care quality and compete in price. Then, various situations are considered. The first corresponds to the case where both insurers do not sign any exclusivity contracts with providers. This situation can be interpreted as a competition model between conventional insurers. Next, we consider a situation where both insurers sign a vertical agreement with one (different) provider, this situation corresponding to a competition between MCOs.

We show that policyholders are better off under a competition between conventional insurers rather than under a managed care competition. This result comes from the fact that under MCOs' competition, the differentiation at the upstream level (between providers) goes down to the insurers' level, allowing them to charge a positive loading factor to their policyholders. Moreover, as it relaxes competition intensity, it induces a lower quality level in equilibrium. It is worth noticing that this insight does not coincide with Gal-Or's (1997) results in which providers are horizontally differentiated, but in an ex post perspective, i.e. ex ante policyholders do not know their preferred health care providers. Indeed, Gal-Or shows that due to the lower prices paid to the providers, policyholders 
get a higher utility under a competition between integrated insurers. Ma (1997) also analyzes the vertical foreclosure strategy with (ex post) differentiated upstream firms while downstream products are sold as option contracts. From the consumers' point of view, Ma reveals that vertical integration consequences are ambiguous and can increase or reduce their welfare.

After that, we continue analyzing the MCOs' competition but this time considering the situation in which one insurer is a mutual, whereas the other one is a private insurer. We reveal the properties of this asymmetric equilibrium. More precisely, we show that due to the nature of its objective, the mutual obtains a higher market share than the private insurer. Providers take advantage of the mutual's objective to increase their mark-up. Moreover, we find that the health care quality provided is higher in the mutual than in the private insurer. Next, we analyze the outcome of the competition between an HMO and a conventional insurer. We reveal that the equilibrium outcome of the game is similar to the preceding equilibrium obtained.

Finally, we analyze the game that determines the market structure that may emerge in equilibrium. Each insurer can choose between an exclusionary and a nonexclusionary strategy. Under the first modality, patients can only obtain health care services from the provider who belongs to their insurer's network. On the contrary, under the second modality, each insurer signs contracts with both providers. Several equilibria may emerge at the outcome of the game. Nevertheless, our results show that the equilibria in which both insurers select one distinct provider, i.e. exclusionary equilibrium - allow them to reach the highest profit level.

It is interesting to analyze this result with respect to the related literature. In an automobile insurance context, Bourgeon et al. (2007) provide an analysis in which some (ex ante) differentiation is present in the providers' market. They also show that an exclusionary equilibrium emerges in equilibrium. Gal-Or (1997) considers a framework in which both levels, i.e. upstream and downstream levels are differentiated. She points out that a nonexclusionary equilibrium arises under the realistic assumption that providers are more differentiated than insurers. On the contrary, when insurers are more differentiated than providers, they both choose an exclusionary strategy. Actually, it is in sharp contrast with our findings. This difference is due to the option value assumption adopted in her framework coming from the ex post horizontal differentiation. Indeed, Gal-Or $(1997,1999)$ considers that ex ante, i.e. under the veil of ignorance, providers deliver health care services perceived as equivalent whereas ex post (after falling ill), each patient has a preferred provider. Due to this, policyholders may suffer from a disutility, calculated in expectation, when their choice among providers is restricted. However, Gal-Or shows that they are better off because it allows insurers to bargain lower health care prices and by consequence, lower premia.

According to the difference in results obtained, it is worth discussing the conceptual difference behind these two assumptions dealing with the providers' horizontal differentiation. This assumption of ex post differentiation suits health care markets particularly well if it is interpreted as providers' specialization 
strategies. For instance, some hospitals can benefit from a better reputation for treating some particular pathologies. ${ }^{1}$ In contrast, when providers' horizontal differentiation has a geographical/distance interpretation, Capps et al. (2003) point out that it is more convincing to consider that patients know ex ante their preferred providers. ${ }^{2}$ It is worth noticing that due to this interpretation of ex ante horizontal differentiation that characterizes providers' competition, our paper may constitute the first analysis to succeed in explaining, by using only strategic interactions arguments, the health care network formation observed in practice. Under the option value approach, Gal-Or shows that health care network could emerged at equilibrium only if insurers were more differentiated than providers, which does not seem to be the most realistic assumption.

In the next section, we present the set-up. Section 3 is devoted to some preliminary considerations dealing with the providers' price-quality competition issue. The comparison between different market structures of the health care sector is exposed section 4 . Section 5 provides an equilibrium analysis. Section 6 concludes.

\section{The set-up}

Three actors are considered in the model:

Policyholders: There is a unit mass of risk averse policyholders, characterized by an initial wealth $w$. They differ by a physical address and are uniformly located over a Hotelling line of interval $[0,1]$. Each policyholder may suffer from a disease with a probability $\pi$. They have preferences described by a twoargument utility function. The first component is a von Neuman-Morgenstern utility function $u$ over his ex post wealth with $u^{\prime}>0$ and $u^{\prime \prime}<0$, the concavity capturing the risk aversion. In case of illness, patients visit a provider $j$ that provides health care of quality $q_{j}$. Then, patients obtain a positive utility $v\left(q_{j}\right)$, with $v^{\prime}>0$ and $v^{\prime \prime}<0$. In words, the quality of health care has a positive impact on patients' health state but with decreasing return. To visit a provider $j$, patients entail a disutility proportional to the distance $t\left|x-x_{j}\right|$. If healthy, policyholders obtain a wealth equal to $w-k_{i}^{j}$, where $k_{i}^{j}$ denotes the premium paid to insurer $i$. In case of illness, patients pay a price $p_{j}$ to provider $j$ and receive an indemnity $s_{i}^{j}+k_{i}^{j}$ from insurer $i$. Their wealth is thus $w+s_{i}^{j}-p_{j}$. Hence, if a policy holder takes out a health insurance contract from insurer $i$ and buys service of quality $q_{j}$ offered by provider $j$ at price $p_{j}$ in case of illness,

\footnotetext{
${ }^{1}$ Moreover, it can be a simple manner to introduce some vertical differentiation between providers. For instance, Baranes and Bardey (2006) and Bardey and Rochet (2010) use this ex post horizontal differentiation argument to introduce some vertical differentiation among insurers and to capture the risk segmentation that occurs between conventional insurers and MCOs or between PPOs and HMOs respectively.

${ }^{2}$ See also Bijslma et al. (2010) in a similar framework and Bardey et al. (2010) dealing with a regulation issue for an opened discussion around the interpretation of horizontal differentiation in providers' market .
} 
his expected utility can be written

$$
E U=(1-\pi) u\left(w-k_{i}^{j}\right)+\pi\left[u\left(w+s_{i}^{j}-p_{j}\right)+v\left(q_{j}\right)-t\left|x-x_{j}\right|\right] .
$$

Providers: Suffering from an illness, policy holders can visit two providers, denoted by $j \in\{0,1\}$. For purpose of simplicity, we assume that each provider has the same cost function $c(q)$ that depends on the quality $q$ delivered with $c^{\prime}>0$ and $c^{\prime \prime} \geq 0$. The two providers are located at the extremities of the segment of length 1 , namely at $x_{0}=0$ and $x_{1}=1$ for providers $j=0$ and $j=1$ respectively. Each provider $j$ 's decides simultaneously on the quality standard $q_{j}$ of health care delivered to patients and sets the price $p_{j}$. The number of patients that visit provider $j$ then depends on the providers' price policies and the insurance contracts. Denoting by $X_{i}^{j}$ the number of policyholders having an insurance contract from insurer $i$ that visit provider $j$, her total demand is thus $\sum_{i} X_{i}^{j}$. Provider $j$ 's objective is given by:

$$
\Phi_{j}=\sum_{i} \phi\left(q_{j}, p_{j}, s_{i}^{j}\right) X_{i}^{j},
$$

under the constraint $p_{j} \geq c\left(q_{j}\right)$, where

$$
\phi(q, p, s)=p-c(q)+\gamma[u(w+s-p)+v(q)]
$$

is the per-patient utility obtained by the provider that depends on two components. The first-one is the net remuneration $p-c(q)$ they receive. The second component denotes the altruism of the providers. Roughly speaking, they put a weight $\gamma$ on their patients' utility. $\gamma=0$ implies that the providers are pure profit maximizers, and of course, we must have $\gamma$ small enough to ensure that $p_{j}>c\left(q_{j}\right)$, which we assume in the following. ${ }^{3}$

Insurers: Two insurers, denoted by $i=\{A, B\}$ offer health plan contracts $\left(k_{i}^{0}, s_{i}^{0}, k_{i}^{1}, s_{i}^{1}\right)$ that depend on the structure of providers' affiliation that we detail in the following. If for example $k_{i}^{0}=k_{i}^{1}=k_{i}$ and $s_{i}^{0}=s_{i}^{1}=s_{i}$ (same insurance contracts with whomever the policy holder wants to visit), the profit function of insurer $i$ is given by:

$$
\Pi_{i}=\left[(1-\pi) k_{i}-\pi s_{i}\right] D_{i},
$$

where $D_{i}$ denotes the total number of policy holders of insurer $i$. More generally, we have

$$
\Pi_{i}=\sum_{j}\left[(1-\pi) k_{i}^{j}-\pi s_{i}^{j}\right] D_{i}^{j} .
$$

The timing of the game is the following:

\footnotetext{
${ }^{3}$ For $\gamma$ large, the provider $j$ 's program is to maximize patients' utility under a break-even constraint, i.e. $\max \left\{u\left(w+s_{i}^{j}-p_{j}\right)+v\left(q_{j}\right): p_{j}-c\left(q_{j}\right) \geq 0\right\}$. Of course, at the optimum we have $p_{j}=c\left(q_{j}\right)$ and the optimal quality level solves $u^{\prime}\left(w+s_{i}^{j}-c\left(q_{j}\right)\right) c^{\prime}\left(q_{j}\right)=v^{\prime}\left(q_{j}\right)$ and thus depends on the health plan net indemnity.
} 
1. Insurers decide on the provider(s) they affiliate. If provider $j$ does not appear in health plan $i$, we adopt the convention that $k_{i}^{j}=s_{i}^{j}=0$.

2. Insurers offer health plans $\left\{k_{i}^{j}, s_{i}^{j}\right\}$ with $i \in\{A, B\}$ and $j \in\{0,1\}$ and simultaneously, providers post prices $p_{j}$ and choose their standard of quality $q_{j}$.

3. Given the observed health plans and providers prices, patients choose at most one insurance.

4. Finally, if sick, policyholders visit a provider among those who are affiliated to their insurer.

\section{Preliminary considerations}

Before comparing different health care organizations, we determine the social optimum from an ex ante and ex post points of view. ${ }^{4}$ Because of policyholders' risk aversion, ex ante, the first-best is characterized by full insurance, i.e. $s=$ $k-p$ against an actuarial premium: $k=\pi c(q)$. Ex post, the socially efficient level of health care quality $q^{F B}$ is solution of

$$
\max _{q} u(w-\pi c(q))+\pi v(q),
$$

and thus solves

$$
v^{\prime}\left(q^{F B}\right)=u^{\prime}\left(w-\pi c\left(q^{F B}\right)\right) c^{\prime}\left(q^{F B}\right) .
$$

The first-best quality level $q^{F B}$ satisfies the equality between the marginal benefit generated by health care and its marginal cost. It is worth noticing that this marginal cost is calculated for an actuarial value of the premium $\pi c\left(q^{F B}\right)$. These considerations are summed-up in the following lemma:

Lemma 1 The first best allocation is characterized by:

i) Full insurance against an actuarial premium $\pi c\left(q^{F B}\right)$.

ii) A level of quality that satisfies: $v^{\prime}\left(q^{F B}\right)=u^{\prime}\left(w-\pi c\left(q^{F B}\right)\right) c^{\prime}\left(q^{F B}\right)$.

The level of quality obtained in the first best and the premium corresponding is crucial to compare and to assess the allocations efficiency corresponding to the different market structures depicted in the next section. Interestingly, we shall see in the following that at an equilibrium where the insurers charge the same premium $k$, providers $j$ ' optimal quality satisfies the condition

$$
v^{\prime}\left(q_{j}\right)=u^{\prime}(w-k) c^{\prime}\left(q_{j}\right),
$$

whatever $\gamma$, the level of the providers' altruism. While the providers' altruism does not explicitly appear in this equation, it influences the nature of the equilibrium; an effect that is embedded in the insurance premium level. A total differentiation of the previous equation gives:

\footnotetext{
${ }^{4}$ See Geoffard (2006) for a similar analysis in an ex post moral hazard context.
} 


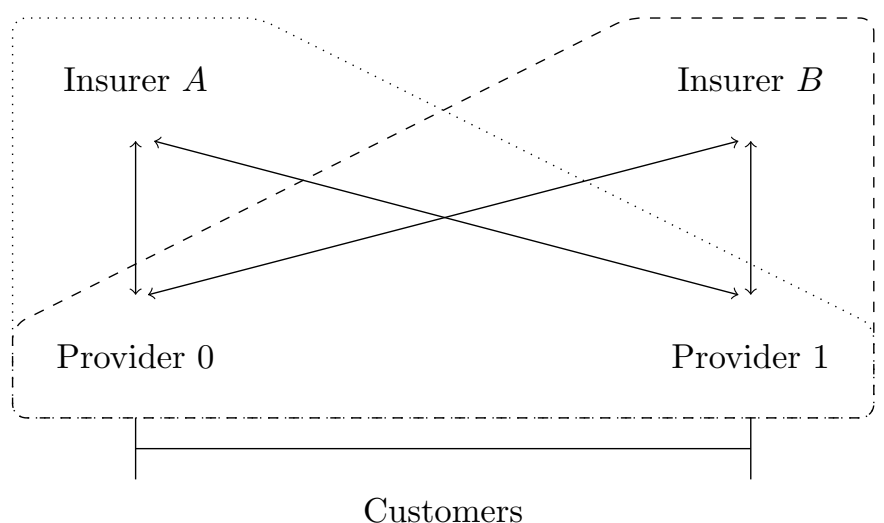

Figure 1: Conventional insurance.

Lemma 2 The level of quality delivered decreases with the insurer's premium.

$$
\frac{d q_{j}}{d k}=-\frac{u^{\prime \prime}(w-k) c^{\prime}\left(q_{j}\right)}{v^{\prime \prime}\left(q_{j}\right)-c^{\prime \prime}\left(q_{j}\right) u^{\prime}(w-k)}<0 .
$$

Hence, if the providers' prices exceed the cost of service, which is typically the case when the altruism coefficient is low, or if insurers apply a positive loading factor on premia, the quality of the health service is lower than the first-best level. More generally, a negative shock on patients' income reduces the quality provided. This result comes from a wealth effect due to the concavity of the utility function (with respect to wealth).

\section{Comparison of different market structures}

In this section, we analyze and compare different affiliation structures. First, we consider a competition between conventional insurers, i.e. insurance companies affiliate both providers: insurer $j$ then charges the same premium $k_{j}^{0}=k_{j}^{1}=k_{j}$ and reimburses the same indemnity $k_{j}+s_{j}$ whatever the provider chosen by the policyholder. Second, we consider the competition between insurers, which adopt an MCO form, i.e. exclusive affiliation of provider. Next, we analyze this market structure when one MCO is a private insurer while the other one is a mutual that maximizes its policyholders' surplus. Finally, we characterize the equilibrium of the game when an MCO competes with a conventional insurer.

\subsection{Conventional insurance}

In the non-exclusive affiliation case, both insurers have affiliated both providers. Figure 1 represents the situation under consideration. 
We directly focus on symmetric equilibria of that subgame in which insurers offer the same policy, i.e. $k_{A}^{j}=k_{B}^{j}=k^{*}$ and $s_{A}^{j}=s_{B}^{j}=s^{*}$ for $j \in\{0,1\}$ while providers offer the same couple price-quality, i.e. $\left(p_{1}, q_{1}\right)=\left(p_{0}, q_{0}\right)=\left(p^{*}, q^{*}\right)$.

\subsubsection{Competition between insurers}

In such a situation, insurers are not differentiated in the sense that policyholders can visit whoever health provider they like in the case they get sick. Hence, insurers compete in a framework of perfect competition. Therefore, competition between insurers leads them to solve the following program:

$\left(k^{*}, s^{*}\right) \in \arg \max _{k, s}\left\{(1-\pi) u(w-k)+\pi\left[u\left(w-p^{*}+s\right)+v\left(q^{*}\right)\right]:(1-\pi) k=\pi s\right\}$.

Introducing the insurers' budget constraint in the objective function leads to:

$$
\left\{\begin{array}{l}
s^{*}=(1-\pi) p^{*} \\
k^{*}=\pi p^{*}
\end{array}\right.
$$

The equilibrium in this subgame involves full insurance for policyholders and actuarial premia.

\subsubsection{Competition between providers}

A proportion $\pi$ of policyholders suffer from a disease and are willing to buy health care treatments offered by providers in the market. Let us denote by $\tilde{x}$ the address over $[0,1]$ that defines the marginal patient who is indifferent between provider 0 and 1 . We have

$$
\tilde{x}=\frac{1}{2}+\frac{1}{2 t}\left[u\left(w-p^{0}+s^{*}\right)+v\left(q^{0}\right)-u\left(w-p^{1}+s^{*}\right)-v\left(q^{1}\right)\right],
$$

and demands for provider 0 and 1 are given by $X^{0}=\pi \tilde{x}$ and $X^{1}=\pi(1-\tilde{x})$ respectively. Provider $j$ 's program is

$$
\left(q^{j}, p^{j}\right) \in \arg \max _{p, q}\left\{\phi\left(q, p, s^{*}\right) X^{j}: p \geq c(q)\right\} .
$$

Assuming that providers are relatively selfish, i.e. $\gamma$ is low enough, we have $p_{j}>c\left(q_{j}\right)$. Therefore, the optimal price and quality delivered by provider 0's, given the health service of provider 1, verify the following first-order conditions:

$$
\left[1-\gamma u^{\prime}\left(w+s^{*}-p_{0}\right)\right] \tilde{x}-\frac{\phi\left(q_{0}, p_{0}, s^{*}\right) u^{\prime}\left(w+s^{*}-p_{0}\right)}{2 t}=0
$$

and,

$$
-\left[c^{\prime}\left(q_{0}\right)-\gamma v^{\prime}\left(q_{0}\right)\right] \tilde{x}+\frac{\phi\left(q_{0}, p_{0}, s^{*}\right) v^{\prime}\left(q_{0}\right)}{2 t}=0 .
$$

Combining these two expressions, we get

$$
v^{\prime}\left(q_{0}\right)=u^{\prime}\left(w-k^{*}\right) c^{\prime}\left(q_{0}\right)
$$

as mentioned above. At the symmetric equilibrium, we obtain: 
Lemma 3 When $\gamma$ is low, i.e. $p^{*}>c\left(q^{*}\right)$, health care price and quality are given by the following system:

$$
v^{\prime}\left(q^{*}\right)=c^{\prime}\left(q^{*}\right) u^{\prime}\left(w-\pi p^{*}\right),
$$

and,

$$
\phi\left(q^{*}, p^{*},(1-\pi) p^{*}\right)=\frac{t\left(1-\gamma u^{\prime}\left(w-\pi p^{*}\right)\right)}{u^{\prime}\left(w-\pi p^{*}\right)} .
$$

Equation (4) reveals that providers obtain some rents in equilibrium due to the horizontal differentiation that characterizes their market. In particular, if providers are selfish, (4) becomes

$$
\phi\left(q^{*}, p^{*},(1-\pi) p^{*}\right)=t / u^{\prime}\left(w-\pi p^{*}\right) .
$$

with the first-best situation at the limit case $t=0$. However, if providers are sufficiently altruistic, i.e. $\gamma$ large enough to have $p=c(q)$, the first-best situation is the result of the market competition even when $t>0$ since insurance premia are actuarially fair. More generally, we have the following result:

Proposition 1 In a symmetric equilibrium $\left(p^{*}, q^{*}, s^{*}, k^{*}\right)$ with conventional insurance:

1. Insurers offer full coverage at actuarial price: $s^{*}=(1-\pi) p^{*}$ and $k^{*}=\pi p^{*}$.

2. If providers are relatively selfish, the price of health service verifies $p^{*}>$ $c\left(q^{*}\right)$, the quality level of health services is lower than the first best value i.e. $q^{*}<q^{F B}$ and the corresponding insurance premium high $k^{*}>k^{F B}$. Otherwise, $q^{*}=q^{F B}$ and $p^{*}=c\left(q^{F B}\right)$.

Proposition 1 says that competition between conventional insurers allows to achieve the ex ante efficiency criterion. When providers are sufficiently altruistic, they also choose the first-best quality level and set a price equal to its marginal cost. On the contrary, if they are more selfish, they obtain a positive mark-up $p^{*}-c\left(q^{*}\right)$ and they choose a quality standard lower than the first best level. Finally, it is worth noticing that in spite of the ex ante providers' differentiation, there is no market failure in a competition between conventional insurers as long as providers are sufficiently altruistic.

\subsection{Competition between MCOs}

Let us now consider the case of exclusive affiliation. Without loss of generality, we consider that insurer $A$ makes a contract with provider 0 while insurer $B$ has an agreement with provider 1 as illustrated in Fig. 2.

Insurers $A$ and $B$ thus verify $\left\{k_{A}^{1}, s_{A}^{1}\right\}=\left\{k_{B}^{0}, s_{B}^{0}\right\}=\{0,0\}$ and, as we focus on a symmetric equilibrium, $\left\{k_{A}^{0}, s_{A}^{0}\right\}=\left\{k_{B}^{1}, s_{B}^{1}\right\}=\{\hat{k}, \hat{s}\}$. 


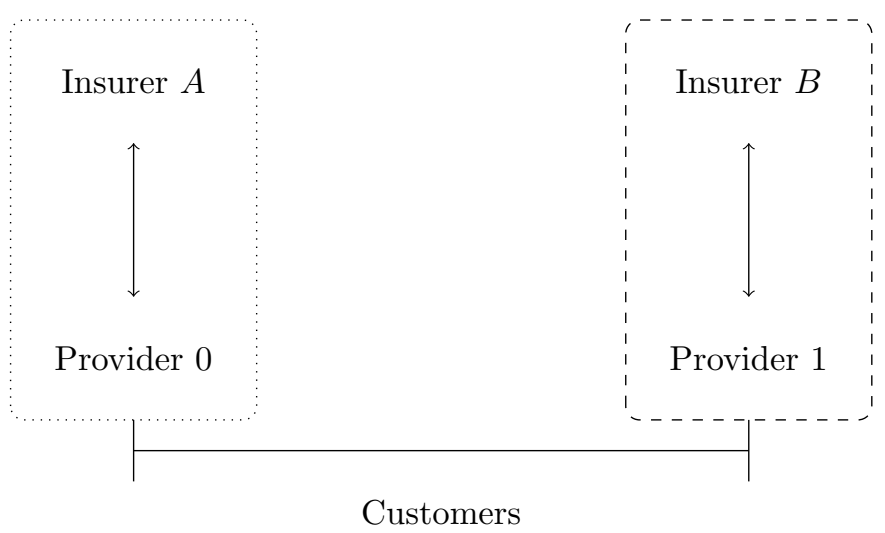

Figure 2: Competition between HMOs.

\subsubsection{Competition in contracts between insurers}

In this framework, patients have to visit the provider affiliated to their insurer. As usual, the horizontal differentiation that characterizes providers' competition drops to the health insurance market. The marginal policyholder, the individual who is indifferent between the two health plans, is located at

$$
\begin{aligned}
\tilde{x}= & \frac{1}{2}+\frac{1}{2 \pi t}\left\{\pi\left[u\left(w+s_{A}^{0}-p_{0}\right)-u\left(w+s_{B}^{1}-p_{1}\right)+v\left(q_{0}\right)-v\left(q_{1}\right)\right]\right. \\
& \left.+(1-\pi)\left[u\left(w-k_{A}^{0}\right)-u\left(w-k_{B}^{1}\right)\right]\right\},
\end{aligned}
$$

which also corresponds to the demand addressed to insurer $A$ (and $1-\tilde{x}$ to insurer $B$ ). Insurer A maximizes its profit:

$$
\left(k_{A}^{0}, s_{A}^{0}\right) \in \arg \max _{k, s}[(1-\pi) k-\pi s] \tilde{x} .
$$

At a symmetric equilibrium, the premium and the indemnity are then characterized as follows:

$$
\left\{\begin{array}{l}
\hat{s}+\hat{k}=\hat{p}, \\
\hat{k}=\pi \hat{p}+\pi t / u^{\prime}(w+\hat{s}-\hat{p}) .
\end{array}\right.
$$

The equilibrium of this subgame indicates that policyholders still benefit from full insurance in spite of the positive loading factor charged by the insurers i.e. ex ante efficiency is still achieved.

\subsubsection{Competition at the providers' level}

At the upstream level, under exclusive agreements, the provider gets a fraction $\pi$ of the insurer who belongs to the same network. Hence, provider 0's problem can be written as

$$
\max _{p, q}\{\phi(q, p, \hat{s}) \pi \tilde{x}: p \geq c(q)\} .
$$


Still assuming that $\gamma$ is low enough, the first-order conditions are similar to (1) and (2). We obtain:

Lemma 4 In a symmetric equilibrium with exclusive affiliation $(\hat{p}, \hat{q}, \hat{s}, \hat{k})$ :

i) Insurers offer full coverage at non actuarial price: $\hat{s}+\hat{k}=\hat{p}$ and $\hat{k}-\pi \hat{p}=$ $\pi t / u^{\prime}(w+\hat{s}-\hat{p})$.

ii) If providers are relatively selfish, the quality and the price of health services verify

$$
v^{\prime}(\hat{q})=c^{\prime}(\hat{q}) u^{\prime}(w-\hat{k}) .
$$

As observed above, the exclusive affiliation structure allows insurers to capture some of the providers' rent since the premia are not actuarial in equilibrium. This result is standard in the vertical integration literature in general, and depicted in Ma (1997) for the health care sector. Even though ex ante insurers are identical, vertical agreements allow them to take advantage of the horizontal differentiation that characterizes the providers' market (embedded in $t>0$ ). The quality of the providers' service follows the same rule as in the non-exclusive affiliation case: it corresponds to the level of quality that maximizes the patients' utility under the constraint of reaching a minimum mark-up level. However, as the premium is no longer actuarially fair, $\hat{k}$ and $k^{*}$ take different values. It affects both the quality and price values. The next proposition compares the allocations obtained in the two affiliation structure, i.e. non-exclusive and exclusive affiliations:

Proposition 2 Compared to non-exclusive affiliation, exclusive affiliation induces:

i) A lower provider price: $\hat{p}<p^{*}$.

ii) A lower health service quality: $\hat{q}<q^{*}$.

iii) An higher premium: $\hat{k}>k^{*}$.

Proof. See appendix.

Consequently, policyholders reach a higher expected utility under non-exclusive affiliation than under exclusive affiliation thanks to a higher quality and a lower premium. On the contrary, providers are worse off because on one hand, they supply a lower quality (the altruism component) and on the other, they benefit from a lower mark-up. Insurers are better off because the exclusive affiliation transfers the providers' differentiation at the downstream level.

Proposition 2 does not signify that in a market where a MCO and a conventional insurer would compete each other, policyholders that would have chosen the conventional insurer would necessarily be better off than policyholders affiliated to the MCO. Proposition 2 only shows that policyholders are better off in a competition between conventional insurers rather than in a competition between MCOs. The mixed duopoly properties are analyzed in the next sub-section. 


\subsection{Competition between two integrated insurers: Mutual vs private $\mathrm{MCO}$}

In this case, we still maintain the exclusive affiliation framework. The only difference concerns the insurers' nature. Without loss of generality, let us consider that insurer $A$ is a mutual while insurer $B$ is a private insurer. We thus consider that $B$ is a profit maximizer while $A$ maximizes his policyholders' surplus subject to a budget constraint.

\subsubsection{Insurers' market}

At the downstream level, insurer A's program is to maximize his policy holders' expected utility under a break-even constraint:

$$
\max _{k_{A}, s_{A}}\left\{(1-\pi) u\left(w-k_{A}\right)+\pi\left[u\left(w-p_{0}+s_{A}\right)+v\left(q_{0}\right)\right]:(1-\pi) k_{A}=\pi s_{A}\right\},
$$

which leads to

$$
\left\{\begin{array}{l}
s_{A}=(1-\pi) p_{0} \\
k_{A}=\pi p_{0}
\end{array}\right.
$$

As insurer $B$ is a profit maximizer, his program is:

$$
\max _{k_{B}, s_{B}}\left[(1-\pi) k_{B}-\pi s_{B}\right](1-\tilde{x}),
$$

where $\tilde{x}$ is defined by (5). The first order conditions lead to:

$$
\left\{\begin{array}{l}
s_{B}+k_{B}=p_{1}, \\
k_{B}=\pi p_{1}+\pi(1-\tilde{x}) 2 t / u^{\prime}\left(w-k_{B}\right) .
\end{array}\right.
$$

Policyholders who choose $B$ thus have full insurance. Nevertheless, insurer $B$ benefits from his provider's market power to charge a positive loading factor in the premium.

Remark 1 Note that if $t=0$, the equilibrium is symmetric.

When $t=0$, each insurer, independently of his objective, maximizes their policyholders' surplus. It leads to a symmetrical equilibrium in which providers and insurers make no profit

\subsubsection{Providers' market}

The providers' programs are unchanged and lead to the following conditions:

$$
\begin{aligned}
& v^{\prime}\left(q_{1}\right)=c^{\prime}\left(q_{1}\right) u^{\prime}\left(w-k_{B}\right), \\
& v^{\prime}\left(q_{0}\right)=c^{\prime}\left(q_{0}\right) u^{\prime}\left(w-k_{A}\right) .
\end{aligned}
$$

Let us define $\tau$ as the loading factor charged by insurer $B$. The following proposition characterizes and compares the slope of control variables i.e. $q$ and $p$, with respect to $\tau$ at the plot $\tau=0$. 
Proposition 3 When $\tau$ is low ( $t$ close to zero) and $c(q)=q$, the following properties hold:

i) Quality increases more in the mutual than in the private insurer: $d q_{0} / d \tau>$ $d q_{1} / d \tau$.

ii) Providers' prices increase more in the mutual than in the private insurer: $d p_{0} / d \tau>d p_{1} / d \tau$.

iii) In the mutual, the provider's mark-up increases $d\left[p_{0}-q_{0}\right] / d \tau>0$.

iv) In the private insurer, the provider's mark-up increases less rapidly than in the mutual $d\left[p_{1}-q_{1}\right] / d \tau<d\left[p_{0}-q_{0}\right] / d \tau$.

v) The mutual's market share increases.

Proof. See appendix.

As we previously pointed out, the situation $t=0$ corresponds to a symmetrical equilibrium in which neither the insurers nor the providers make profits. Proposition 3 allows us to characterize some properties of the asymmetric equilibrium, i.e. what would happen in the neighborhood of this symmetric equilibrium as long as the differentiation increases at the upstream level. First, due to the fact that it does not charge a positive loading factor, the mutual increases his market share (v). Concerning the providers, we have two effects at work: On the one hand, they seek to increase the quality provided for a given price. On the other hand, for a given quality level, they set an higher price to increase their profits. Because of the asymmetry between the insurers, the resulting effects are in favor of the provider affiliated to the mutual (provider 0) who takes advantage of the higher market share of the mutual: she provides a higher quality than provider 1 in equilibrium (i), she charges an higher price (ii) which allow her to increase her mark-up more heavily than provider 1 (points iii and iv).

\subsection{MCO vs conventional insurer competition}

The last case considered occurs when an insurer, say $A$, affiliates only one provider, say 0 , while $B$ affiliates both providers. We go back to the previous assumption and consider that they are both profit maximizers. This situation is represented in the following figure.

Because the two insurers offer a health plan for provider 0 , competition is fierce and results in health plans with complete insurance at actuarially fair premia for this provider. Then, we have

$$
k_{A}=k_{B}^{0}=\pi p_{0}
$$

and,

$$
s_{A}=s_{B}^{0}=(1-\pi) p_{0},
$$


and no insurer extracts a rent from provider's 0 health plan. In contrast, individuals who choose provider 1 are all insured by insurer $B^{5}$. Hence, the situation in term of insurance plans is similar to the MCO vs mutual case analyzed above: the expression of the indifferent consumer's location is the same as above and insurer $B$ determines his offer $\left(k_{B}^{1}, s_{B}^{1}\right)$ for provider 1 by solving:

$$
\max _{k_{B}, s_{B}}\left[(1-\pi) k_{B}-\pi s_{B}\right](1-\tilde{x}) .
$$

It is thus apparent that this situation results in the same insurance plans and providers' prices and qualities as above, because the competition between insurers on provider 0 results in the same plan as the one offered by a mutual and also because insurer $B$ has an exclusive link with provider 1 .

\section{Market structure}

In the previous section, all the possible affiliation structures have been reviewed. Following Gal-Or (1997), we can now determine whether some of them are more likely than others to emerge by considering the first stage of the game: at the very beginning, before insurers and providers make their offer, insurers choose non-cooperatively to affiliate one or both providers. Denote by $\hat{R}$ the insurer profit under exclusive affiliation, i.e. $\hat{R}=[(1-\pi) \hat{p}-\hat{s}] / 2$. As shown in the previous subsection, the profit of the insurance company which affiliates both providers in the asymmetric affiliation case is strictly smaller than $\hat{R}$ : he makes no profit on the insureds who have chosen the provider shared with his rival, and due to this fierce competition, the market share on his exclusive provider is reduced. To reduce the notational burden, his profit in that case is denoted by $\hat{R}-\eta$ (with $\hat{R}>\eta>0$ ) without loss of generality.

For the sake of clarity, the payoffs for the insurers in the different configurations are summarized in Table 1.

\begin{tabular}{|c|c|c|c|}
\hline $\begin{array}{ll}\text { Insurer A } & \text { Insurer B } \\
\end{array}$ & Provider 0 & Provider 1 & Provider $0 \& 1$ \\
\hline Provider 0 & $(0,0)$ & $(\hat{R}, \hat{R})$ & $(0, \hat{R}-\eta)$ \\
\hline Provider 1 & $(\hat{R}, \hat{R})$ & $(0,0)$ & $(0, \hat{R}-\eta)$ \\
\hline Provider 0 \& 1 & $(\hat{R}-\eta, 0)$ & $(\hat{R}-\eta, 0)$ & $(0,0)$ \\
\hline
\end{tabular}

Table 1: Insurers' payoffs depending on the affiliation choices.

A look at this table shows that there are tree Nash equilibria of this affiliation game: two of them correspond to exclusive affiliation ((Provider 0, Provider

\footnotetext{
${ }^{5}$ We have also explored the situation in which insurer $B$ affiliates both providers but offer a unique insurance contract containing cross-subsidies. However, in such content, there is no equilibrium.
} 
1) and (Provider 1, Provider 0)), while (Provider 0\&1, Provider 0\&1) is the conventional insurance situation. As the exclusive affiliation cases correspond to the higher profits levels, we have the following result:

Proposition 4 The affiliation game has three Nash equilibria: the two exclusive affiliation structures and the non-exclusive affiliation structure. From the insurers' standpoint, the exclusive affiliation equilibria Pareto-dominate the nonexclusive affiliation equilibrium.

If insurance insurer $A$ decides to affiliate exclusively one provider, it is an optimal decision for the rival insurance insurer $B$ to affiliate exclusively the remaining provider: indeed, choosing not to compete with the segment of patients who visit provider 0 softens the competition between providers and allows insurer $B$ to raise his profit.

\section{Conclusion}

The model provided allows us to point out several results dealing with the network issue in health care markets. The first part of the model is devoted to the comparison of policyholders' welfare according to the market structure considered. First, we show that policyholders' welfare is better off under a competition between conventional insurers rather than under a competition between MCOs. Second, it emphasizes that the competition between two MCOs, one private insurer and one mutual, leads to a similar equilibrium than a competition between a conventional insurer and a MCO.

The last part of the paper characterizes the market structure endogenously. We show that exclusive affiliation structures emerge in the equilibrium of the game. This result is in sharp contrast with previous findings that deal with this issue. We identify that this difference is due to the providers' horizontal differentiation assumption. In this paper, we consider that policyholders' preferences, according to the set of providers, are determined ex ante and not ex post. If the two assumptions make sense and correspond to different aspects of the horizontal dimension, it is interesting to note that only an ex ante horizontal differentiation framework explains the formation of health care networks that we observe in practice.

This paper could be extended in different ways. First, as considered in Bijlsma et al. (2010), it would be worth analyzing the impact of the equilibrium when a positive mass consumers remain uninsured. It could change the nature of the equilibrium and modify the conclusion in terms of welfare comparisons. Second, according to the fact that the results obtained in the literature are sharply different, it would be interesting to consider a bi-dimensional horizontal differentiation, i.e. ex ante as in the present paper and ex post, as in Gal-Or (1997, 1999) and Ma (2002) in order to characterize some different regions of equilibria obtained according to the set of parameters. Finally, it would be useful to consider the policyholders and the providers' segmentation among the 
insurers. It would allow to make the bridge with a two-sided market perspective, as it is considered in Bardey and Rochet (2010).

\section{References}

[1] Arrow KJ., 1963, "Uncertainty and the welfare economics of medical care", American Economic Review, 53(5): 941-973.

[2] Baker L.C. and Corts K., 1996, "HMO Penetration and the Cost of Health Care : Market Discipline vs Market Segmentation", American Economic Review, vol 86, n², p389-394.

[3] Baranes E. and D. Bardey, 2008, "Health Care Markets and Vertical Restraints", Working Paper GREMAQ.

[4] Bardey D. and J-C. Rochet, 2010, "Competition among Health Plans: A Two-Sided Market Approach", Journal of Economics and Management Strategy, Blackwell Publishing, vol. 19(2), 435-451, 06.

[5] Bardey D., Canta C. and J-M. Lozachmeur, 2010, "A New Rationale for Cost Reimbursement: Price Regulation with Horizontal and Vertical Differentiation", Working Paper IDEI.

[6] Bijlsma M., J. Boone and G. Zwart, 2010, "Selective contracting and foreclosure in health care markets", CPB Discussion Paper $n^{\circ} 140$.

[7] Blendon R., M. Brodie, J. Benson, D. Altman, L. Levitt, T. Hoff, and L. Hugick. 1998. "Understanding the Managed Care Backlash", Health Affairs, 17(4):80-94.

[8] Bourgeon J-M., Picard P. and Pouyet J., 2007, "Providers Affiliation, Insurance and Collusion", Journal of Banking and Finance, vol. 32(1), 170-186.

[9] Capps, C., D. Dranove and M. Satterthwaite, 2003, Competition and market power in option demand markets, Rand Journal of Economics, vol. 4, no. $4,737-763$.

[10] Choné P and C.A Ma, 2010, "Optimal Health Care Contracts under Physician Agency", Annales d'Economie et de Statistiques, forthcoming.

[11] Gal-Or E., 1997, "Exclusionary Equilibria in Health Care Markets", Journal of Economics and Management Strategy, vol.6, iss. 1, 5-43.

[12] Gal-Or E., 1999, "Mergers and Exclusionary Practices in Health Care Markets", Journal of Economics and Management Strategy, vol.8(3), 315-350.

[13] Geoffard P-Y., 2006, "Incentive and Selection Effects in Health Insurance", chap. 10 in Elgar Companion to Health Economics, Andrew Jones, Ed. 
[14] Ma C. A., 1994, "Health Care Payment Systems: Cost and Quality Incentives", Journal of Economics 83 Management Strategy, 3, 93-112.

[15] Ma C. A., 1997, "Option Contracts and Vertical Foreclosure", Journal of Economics and Management Strategy, 1997, v. 6, iss. 4, 725-53.

[16] Ma C.A and T. McGuire, 1997, "Optimal Health Insurance and Provider Payment", American Economic Review, vol. 87, issue 4, 685-704.

[17] Ma C-A and M. Riordan, 2002, "Health Insurance, Moral Hazard and Managed Care", Journal of Economics and Management Strategy, vol 11, $\mathrm{n}^{\circ} 1,81-107$.

\section{Appendix}

\section{A Proof of Proposition 2}

The levels of quality supplied in each affiliation case satisfy $\hat{q}=Q(\hat{k})$ and $q^{*}$ $=Q\left(k^{*}\right)$ where $Q(k)$ is the function implicitly defined by

$$
v^{\prime}(Q)=c^{\prime}(Q) u^{\prime}(w-k) .
$$

A total differentiation gives $Q^{\prime}(k)=c^{\prime} u^{\prime \prime} /\left[c^{\prime \prime} u^{\prime}-v^{\prime \prime}\right]<0$, implying that $\hat{q}<q^{*}$ if $\hat{k}>k^{*}$. As $\hat{k}>\pi \hat{p}$, let us denote $\hat{k}=\hat{\tau} \pi \hat{p}$ where $\hat{\tau}>1$ and recall that $k^{*}=\pi p^{*}$. The providers' first-order conditions can be written as $\psi_{\gamma}(\hat{p}, \tau)=t=\psi_{\gamma}\left(p^{*}, 1\right)$ where

$$
\psi_{\gamma}(p, \tau) \equiv\{p-c(Q(\tau \pi p))+\gamma[u(w-\tau \pi p)+v(Q(\tau \pi p))]\} \frac{u^{\prime}(w-\tau \pi p)}{1-\gamma u^{\prime}(w-\tau \pi p)} .
$$

Consider the case $\gamma=0$, i.e. $\psi_{0}(p, \tau) \equiv[p-c(Q(\tau \pi p))] u^{\prime}(w-\tau \pi p)$. We have

$$
\frac{\partial \psi_{0}(p, \tau)}{\partial p}=\left[1-c^{\prime}(Q) Q^{\prime}(\tau \pi p) \tau \pi\right] u^{\prime}(w-\tau \pi p)-[p-c(Q)] u^{\prime \prime}(w-\tau \pi p) \tau \pi>0
$$

and,

$$
\frac{\partial \psi_{0}(p, \tau)}{\partial \tau}=-c^{\prime}(Q) Q^{\prime}(\tau \pi p) \pi p u^{\prime}(w-\tau \pi p)-[p-c(Q)] u^{\prime \prime}(w-\tau \pi p) p \pi>0 .
$$

We thus have $\psi_{0}(\hat{p}, 1)<\psi_{0}(\hat{p}, \hat{\tau})=\psi_{0}\left(p^{*}, 1\right)$ implying $\hat{p}<p^{*}$. Now, suppose that $\hat{k} \leq k^{*}$ which implies $\hat{q} \geq q^{*}$ and since $u(\cdot)$ is concave, $u^{\prime}\left(w-k^{*}\right) \geq u^{\prime}(w-\hat{k})$. As $\hat{p}<p^{*}$, we have $\hat{p}-c(\hat{q})<p^{*}-c\left(q^{*}\right)$ and thus $t=[\hat{p}-c(\hat{q})] u^{\prime}(w-\hat{k})<$ $\left[p^{*}-c\left(q^{*}\right)\right] u^{\prime}\left(w-k^{*}\right)=t$, hence a contradiction. We thus have $\hat{k}>k^{*}$ and $\hat{q}<q^{*}$. As $\psi_{\gamma}(p, \tau)$ is continuous in $\gamma$ for $\gamma$ close to zero, these results are not challenged for $\gamma>0$ as long as $\gamma$ is small. Observe that these results are not challenged when providers are very altruistic. Indeed, we have $p^{*}=c\left(q^{*}\right)$ with $q^{*}$ solving $\chi\left(q^{*}, 1\right)=0$ and $\hat{p}=c(\hat{q})$ with $\chi(\hat{q}, \hat{\tau})=0$ where $\chi(q, \tau) \equiv q-Q(\tau \pi c(q))$. As 


$$
\frac{\partial \chi(q, \tau)}{\partial q}=1-Q^{\prime}(\tau \pi c(q)) \tau \pi c^{\prime}(q)>0
$$

and,

$$
\frac{\partial \chi(q, \tau)}{\partial \tau}=-Q^{\prime}(\tau \pi c(q)) \pi c(q)>0
$$

it comes $\chi(\hat{q}, 1)<\chi(\hat{q}, \hat{\tau})=\chi\left(q^{*}, 1\right)$ implying $\hat{q}<q^{*}$, and thus $p^{*}>\hat{p}$. $q^{*}$ maximizes $u\left(w+s^{*}-c(q)\right)+v(q)$ leading to $u^{\prime}\left(w-k^{*}\right)=v^{\prime}\left(q^{*}\right) / c^{\prime}\left(q^{*}\right)$ and $\hat{q}$ maximizes $u(w+\hat{s}-c(q))+v(q)$. As

$$
\frac{d}{d q}\left[\frac{v^{\prime}(q)}{c^{\prime}(q)}\right]=\frac{v^{\prime \prime}(q) c^{\prime}(q)-v^{\prime}(q) c^{\prime \prime}(q)}{c^{\prime}(q)^{2}}<0
$$

the concavity of $u(\cdot)$ implies $u^{\prime}(w-\hat{k})=v^{\prime}(\hat{q}) / c^{\prime}(\hat{q})>v^{\prime}\left(q^{*}\right) / c^{\prime}\left(q^{*}\right)=u^{\prime}\left(w-k^{*}\right)$, and thus $\hat{k}>k^{*}$.

\section{B Proof of Proposition 4}

Consider first the case of very altruistic providers, implying $p_{j}=c\left(q_{j}\right)$. As $k_{0}=\pi p_{0}$ and $k_{1}=\tau \pi p_{1}$ with $\tau>1$, the quality levels are given by $q_{0}=h(1)$ and $q_{1}=h(\tau)$ where $h(\tau)$ is the function implicitly defined by

$$
v^{\prime}(h)=c^{\prime}(h) u^{\prime}(w-\tau \pi c(h)) .
$$

Differentiating gives $h^{\prime}(\tau)=u^{\prime \prime} \pi c c^{\prime} /\left[-u^{\prime \prime} \tau \pi\left(c^{\prime}\right)^{2}+u^{\prime} c^{\prime \prime}-v^{\prime \prime}\right]<0$, implying that $q_{1}<q_{0}$ hence $p_{1}<p_{0}$. As $q_{j}=Q\left(k_{j}\right)$ with $Q^{\prime}(k)<0$ (see above), we also have $k_{1}>k_{0}$.

Consider now the case of selfish providers, i.e., $\gamma=0$. The first-order conditions of the providers' programs simplify to

$$
\begin{aligned}
\tilde{x} & =\frac{\left[p_{0}-c\left(q_{0}\right)\right] u^{\prime}\left(w-k_{A}\right)}{2 t}, \\
1-\tilde{x} & =\frac{\left[p_{1}-c\left(q_{1}\right)\right] u^{\prime}\left(w-k_{B}\right)}{2 t},
\end{aligned}
$$

where $k_{A}=\pi p_{0}$ and $k_{B}=\tau \pi p_{1}$ with $\tau>1$. Adding these two equations gives, using $c(q)=q$,

$$
2 t=\left[p_{0}-c\left(q_{0}\right)\right] u^{\prime}\left(w-\pi p_{0}\right)+\left[p_{1}-c\left(q_{1}\right)\right] u^{\prime}\left(w-\tau \pi p_{1}\right) .
$$

Using (5) and (6), we also obtain

$$
\pi\left(p_{0}-q_{0}\right) u^{\prime}\left(w-\pi p_{0}\right)=\pi t+\pi\left[v\left(q_{0}\right)-v\left(q_{1}\right)\right]+u\left(w-\pi p_{0}\right)-u\left(w-\tau \pi p_{1}\right) .
$$

Differentiating these two equations yields

$$
d p_{0}\left(u_{0}^{\prime}-\pi u_{0}^{\prime \prime} m_{0}\right)-d q_{0} u_{0}^{\prime}+d p_{1} v_{1}^{\prime}+d q_{1}\left(-v_{1}^{\prime}+v_{1}^{\prime \prime} m_{1}\right)=0
$$




$$
d p_{0}\left(2 u_{0}^{\prime}-\pi u_{0}^{\prime \prime} m_{0}\right)-2 u_{0}^{\prime} d q_{0}-\tau u_{1}^{\prime} d p_{1}+v_{1}^{\prime} d q_{1}=p_{1} u_{1}^{\prime} d \tau,
$$

where $m_{j}=p_{j}-q_{j}, j \in\{0,1\}$.

Differentiating $v^{\prime}\left(q_{i}\right)=c^{\prime}\left(q_{i}\right) u^{\prime}\left(w-k_{j}\right)$ for each provider-insurer pair gives

$$
\begin{aligned}
\tau \pi u_{1}^{\prime \prime} d p_{1}+v_{1}^{\prime \prime} d q_{1} & =-p_{1} \pi u_{1}^{\prime \prime} d \tau \\
\pi u_{0}^{\prime \prime} d p_{0}+v_{0}^{\prime \prime} d q_{0} & =0 .
\end{aligned}
$$

We thus obtain the following system

$$
\left[\begin{array}{l}
d p_{0} / d \tau \\
d q_{0} / d \tau \\
d p_{1} / d \tau \\
d q_{1} / d \tau
\end{array}\right]=p_{1} u_{1}^{\prime} A(\tau)^{-1}\left[\begin{array}{c}
0 \\
1 \\
-\pi u_{1}^{\prime \prime} / u_{1}^{\prime} \\
0
\end{array}\right]
$$

where

$$
A(\tau)=\left[\begin{array}{cccc}
u_{0}^{\prime}-\pi u_{0}^{\prime \prime} m_{0} & -u_{0}^{\prime} & v_{1}^{\prime} & -v_{1}^{\prime}+v_{1}^{\prime \prime} m_{1} \\
2 u_{0}^{\prime}-\pi u_{0}^{\prime \prime} m_{0} & -2 u_{0}^{\prime} & -\tau u_{1}^{\prime} & v_{1}^{\prime} \\
0 & 0 & \tau \pi u_{1}^{\prime \prime} & v_{1}^{\prime \prime} \\
\pi u_{0}^{\prime \prime} & v_{0}^{\prime \prime} & 0 & 0
\end{array}\right]
$$

When $t \rightarrow 0$, we have $\tau \rightarrow 1$ and $p_{1} \rightarrow p_{0}, q_{1} \rightarrow q_{0}$ and the same for the marginal utility levels. Hence, using $v_{0}^{\prime}=u_{0}^{\prime}$ we get

$$
\begin{aligned}
\Delta & \equiv \operatorname{det}(A(1))=\pi^{2} u_{0}^{\prime \prime 2}\left[m_{0} v_{0}^{\prime \prime}\left(4 v_{0}^{\prime}-m_{0} v_{0}^{\prime \prime}\right)-3 u_{0}^{\prime 2}\right]-2 \pi v_{0}^{\prime \prime} u_{0}^{\prime \prime} u_{0}^{\prime}\left(3 u_{0}^{\prime}-2 m_{0} v_{0}^{\prime \prime}\right)-3 u_{0}^{\prime 2} v_{0}^{\prime \prime 2} \\
& <0
\end{aligned}
$$

and we obtain

$$
\begin{gathered}
\left.\frac{d p_{0}}{d \tau}\right|_{\tau=1}=-p_{0} v_{0}^{\prime 2} v_{0}^{\prime \prime}\left(\pi u_{0}^{\prime \prime}+v_{0}^{\prime \prime}\right) / \Delta>0, \\
\left.\frac{d q_{0}}{d \tau}\right|_{\tau=1}=-\left.\pi \frac{u_{0}^{\prime \prime}}{v_{0}^{\prime \prime}} \frac{d p_{0}}{d \tau}\right|_{\tau=1}<0 \\
\left.\frac{d p_{1}}{d \tau}\right|_{\tau=1}=\left\{u_{0}^{\prime \prime 2} v_{0}^{\prime \prime 2}+\pi u_{0}^{\prime} u_{0}^{\prime \prime} v_{0}^{\prime \prime}\left(4 u_{0}^{\prime}-3 v_{0}^{\prime \prime} m_{0}\right)+\pi^{2} u_{0}^{\prime \prime 2}\left[u_{0}^{\prime}\left(3 v_{0}^{\prime}-4 v_{0}^{\prime \prime} m_{0}\right)+v_{0}^{\prime \prime} v_{0}^{\prime \prime} m_{0}^{2}\right]\right\} p_{0} / \Delta \\
<0,\left.\quad \frac{d q_{1}}{d \tau}\right|_{\tau=1}=p_{0} \pi u_{0}^{\prime \prime} v_{0}^{\prime}\left[2 u_{0}^{\prime} v_{0}^{\prime \prime}+\pi u_{0}^{\prime \prime}\left(2 u_{0}^{\prime}-m_{0} v_{0}^{\prime \prime}\right)\right] / \Delta<0, \\
\left.\frac{d q_{1}}{d \tau}\right|_{\tau=1}-\left.\frac{d q_{0}}{d \tau}\right|_{\tau=1}=\frac{p_{0} \pi u_{0}^{\prime \prime} v_{0}^{\prime}}{\Delta}\left[2 u_{0}^{\prime} v_{0}^{\prime \prime}+\pi u_{0}^{\prime \prime}\left(2 u_{0}^{\prime}-m_{0} v_{0}^{\prime \prime}\right)-\left(\pi u_{0}^{\prime \prime} v_{0}^{\prime}+u_{0}^{\prime} v_{0}^{\prime \prime}\right)\right] \\
=\frac{p_{0} \pi u_{0}^{\prime \prime} v_{0}^{\prime}}{\Delta}\left(u_{0}^{\prime} v_{0}^{\prime \prime}+\pi u_{0}^{\prime \prime} u_{0}^{\prime}-\pi u_{0}^{\prime \prime} m_{0} v_{0}^{\prime \prime}\right)<0 .
\end{gathered}
$$


We have $\left.\left(d m_{0} / d \tau\right)\right|_{\tau=1}>0$ and

$$
\left.\frac{d m_{1}}{d \tau}\right|_{\tau=1}-\left.\frac{d m_{0}}{d \tau}\right|_{\tau=1}=\left\{2 u_{0}^{\prime} v_{0}^{\prime \prime 2}+\pi u_{0}^{\prime \prime} v_{0}^{\prime \prime}\left(4 u_{0}-3 m_{0} v_{0}^{\prime \prime}\right)+\pi^{2} u_{0}^{\prime \prime 2}\left(2 u_{0}^{\prime}-3 m_{0} v_{0}^{\prime \prime}+m_{0}^{2} v_{0}^{\prime \prime 2} / u_{0}^{\prime}\right)\right\} / \Delta<0 .
$$

Using (6), we have

$$
\tilde{x}=\frac{m_{0} u^{\prime}\left(w-\pi p_{0}\right)}{m_{1} u^{\prime}\left(w-\tau \pi p_{1}\right)+m_{0} u^{\prime}\left(w-\pi p_{0}\right)} .
$$

Differentiating yields

$$
\left.\frac{d \tilde{x}}{d \tau}\right|_{\tau=1}=\frac{\left.3\left[\left(d m_{0} / d \tau\right) u_{0}^{\prime}-m_{0} u_{0}^{\prime \prime} \pi\left(d p_{0} / d \tau\right)\right]\right|_{\tau=1}-\left.\left[\left(d m_{1} / d \tau\right) u_{1}^{\prime}-m_{1} u_{1}^{\prime \prime} \pi\left(p_{1}+\tau d p_{1} / d \tau\right)\right]\right|_{\tau=1}}{4 m_{0} u_{0}^{\prime}}
$$

where,

$$
\left.\left[\frac{d m_{1}}{d \tau} u_{1}^{\prime}-m_{1} u_{1}^{\prime \prime} \pi\left(p_{1}+\tau \frac{d p_{1}}{d \tau}\right)\right]\right|_{\tau=1}=u_{0}^{\prime 2}\left(\pi u_{0}^{\prime \prime}+v_{0}^{\prime \prime}\right)\left[u_{0}^{\prime} v_{0}^{\prime \prime}+\pi u_{0}^{\prime \prime}\left(u_{0}^{\prime}-m_{0} v_{0}^{\prime \prime}\right)\right] p_{0} / \Delta<0
$$

implying $\left.(d \tilde{x} / d \tau)\right|_{\tau=1}>0$. 